\title{
Questions on Optimization of Measurement of Total Electron Content in Ionosphere with GPS
}

\author{
Arif Shafayat Mehdiyev1, Ramiz Ahmed Eminov', Hikmat Hamid Asadov3, \\ Natig Hajiaga Javadov 4 \\ ${ }^{1}$ National Academy of Aviation, Baku, Azerbaijan \\ ${ }^{2}$ State Oil Academy, Baku, Azerbaijan \\ ${ }^{3}$ Research Institute of Aerospace Informatics, Baku, Azerbaijan \\ ${ }^{4}$ Industrial Association Promavtomatika, Baku, Azerbaijan \\ Email: asadzade@rambler.ru
}

Received 28 May 2014; revised 25 June 2014; accepted 25 July 2014

Copyright (C) 2014 by authors and Scientific Research Publishing Inc.

This work is licensed under the Creative Commons Attribution International License (CC BY).

http://creativecommons.org/licenses/by/4.0/

c) (i) Open Access

\begin{abstract}
The analysis of existing method for calculation of total content of electrons (TEC) in ionosphere using GPS occultation method does show that due to different values of signal/noise ration in GPS signals $L_{1}$ and $L_{2}$, the new method of optimum measurements of relevant frequency components of TEC measured by phase and code methods should be developed. The optimum quantity of measurements of the above-mentioned frequency components is determined taking into account the limitation imposed on general number of necessary measurements.
\end{abstract}

\section{Keywords}

GPS Receiver, Ionosphere, Optimization, Total Electron Content, Measurements

\section{Introduction}

It is well-known that the measurements of total electron content (TEC) and scintillation parameters of ionosphere can be carried out with GPS which is important for study of this layer of atmosphere.

The measurement of said parameters of ionosphere was carried out during last 20 years, but accuracy of such measurements was not so high. Increase of accuracy of said measurements is important from the view point of modification of monitoring of ionosphere and is vital for study of non-stability and turbulence of ionospheric plasma. 
As it is noted in the work [1], the distortions of results of GPS measurements caused by ionosphere factors are one of the most significant components of total error of positioning using GPS. Delay of GPS signals due to effect of ionospheric electrons can be featured and taken account by carrying out double frequency measurements, signed as GPS $L_{1}$ and $L_{2}$.

\section{Critical Review of Existing Methods}

According to [1], if the frequency of the GPS signal too exceeds the frequency of ionospheric plasma, the group delay $\Delta r$ measured by meters, should be defined as:

$$
\Delta r=\frac{40.3}{f^{2}} \cdot T E C,
$$

where TEC—-total electronic content (electrons per square meter); $f$-frequency of GPS signal.

It should be noted, that one unit of TEC, equal to $10^{16}$ electron $\cdot \mathrm{m}^{-2}$ causes delay of $L_{1}$ equal to $0.163 \mathrm{~m}$, and $L_{2}-0.267 \mathrm{~m}$.

According to [1], the following interrelation of major parameters used in double frequency measurements of TEC does exist,

$$
T E C_{\rho}=\frac{\rho_{L_{1}}-\rho_{L_{2}}}{0.104 m T E C U^{-1}},
$$

where $T E C U=10^{16}$ electron $\cdot \mathrm{m}^{-2} ; \quad \rho_{L_{1}}$ and $\rho_{L_{2}}$-measured pseudo ranges.

It should be noted, that the Formula (2) is true for carrying out of GPS code measurements.

As it is noted in [1], the total electron content in ionosphere also can be determined by carrying out the measurements of phase of carrier signal of $L_{1}$ and $L_{2}$. If that, the following formula is true,

$$
T E C_{\varphi}=\frac{-\varphi_{L_{1}}+\varphi_{L_{2}}}{0.104 m T E C U^{-1}},
$$

where $\varphi_{L_{1}}$ and $\varphi_{L_{2}}$-phases of signals $L_{1}$ and $L_{2}$, transformed to ranges.

In order to calculate the resulting values of $T E C_{f}$ the following algorithm is suggested in [1]:

The averaged value of difference $T E C(t)_{\rho}-T E C(t)_{\varphi}$ as a function of time should be calculated using least squares method,

$$
T E C_{\delta}=\text { l.s.m. }\left[T E C(t)_{\rho}-T E C(t)_{\varphi}\right] .
$$

The final value of difference $T E C_{f}$ should be determined as:

$$
T E C_{f}=T E C_{\varphi}-T E C_{\delta} .
$$

According to [1], the above described method allows to calculate the smoothed and absolute amount of $T E C_{\delta}$ in link with each satellite during whole time of its functioning.

At the same time, such method of calculation of TEC in ionosphere is non-effective due to following reasons. As it is noted in the work [2], the disperse nature of ionosphere causes the temporal delay inversely proportional to square of frequency of radio signal. This, in principle, allows removing the ionospheric error during geodetic GPS measurements carrying out combinational double frequency method of positioning. In this case, the above said dispersive property is strictly observed in the Formula (1). According to this formula the group delay should be more at the frequency $L_{2}$ (we remind, that $L_{1}=1.575 \mathrm{GHs} ; L_{2}=1.227 \mathrm{GHs}$ ).

In line with works [3]-[5], the signal/noise ratios for frequencies $L_{1}$ and $L_{2}$ are sharply different. Upon use of radio occultation method the signal/noise ratios of received GPS signals are not constant temporally due to defocusing and/or attenuation of radio signal. The difference between signal/noise ratios for $L_{1}$ and $L_{2}$ frequencies is not less than $15 \%$ - $20 \%$ and can reach $100 \%$ and more.

Taking into account such a significant difference between signal/noise ratios for $L_{1}$ and $L_{2}$, the method described in [1], based on of Formulas (2)-(5), can be considered as non-rational, because this method provides for carrying out of joint statistical processing of signal featured by different level noisiness.

Authors of the work [6] suggest taking into account the different level of noises in $L_{1}$ and $L_{2}$ signals in 
GPS occultation measurements by calculating the linear combination of these signals. It is noted, that custom linear combination of $L_{1}$ and $L_{2}$ is functioning well only if noises are miserable. But, in reality the quality of signal $L_{1}$ is more higher, than that of $L_{2}$, which commonly is not taken into account in such linear combinations.

Authors of [6] suggest to develop such a combination of signals, which would take into account the different levels of their noisiness. In this case $L_{1}$ and $L_{2}$ should be processed independently each other. The weight coefficients of these components accord to their estimated dynamic accuracy. It is noted, that such a method allows increasing the total effectiveness of radio occultation method.

\section{Optimization of Measuring of TEC in Ionosphere Using GPS}

The purpose of the present article is further development of idea, suggested in [6] concerning independent processing of $L_{1}$ and $L_{2}$ signals by way of optimization of the measurement process using the limitation condition imposed on general resources of measuring operations.

As it was noted above, in line with the method, suggested in [1], the average value of $T E C(t)_{p}$ and

$\operatorname{TEC}(t)_{\varphi}$ should be calculated using formula [4]. In its turn, to calculate the average value of $\operatorname{TEC}(t)_{p}$, in line with Formula (2), the average values of $\rho_{L_{1}}$ and $\rho_{L_{2}}$ should be calculated.

But it is well-known that upon calculation of averaged values of noisy measuring signals the random error of measurement decreases by $\sqrt{n}$ times, where $n$-number of measurements.

The task of optimal organization of measurement upon realization of above described method can be formulated as follows. Assume, that the limitation condition is imposed on total number amount of carried out measurement operations by purpose to calculate the average values of $\rho_{L_{1}}$ and $\rho_{L_{2}}$, i.e. the number of measurement operations is limited and equal to $n_{0}$.

Let us signify the random error of measurement of $\rho_{L_{1}}$ as $\sigma_{L_{1}}$ and $\rho_{L_{2}}$ as $\sigma_{L_{2}}$.

The total random error of measuring of $T E C(t)_{p}$ can be determined as:

$$
\sigma_{T E C}^{2}=\sigma_{L_{1}}^{2}+\sigma_{L_{2}}^{2} .
$$

If we assume, that $L_{1}$ should be measured by $n_{1}$ times, then $L_{2}$ may be measured by $n_{0}-n_{1}$ times. Therefore, the total random error can be determined as:

$$
\sigma_{T E C_{0}}^{2}=\frac{\sigma_{L_{1}}^{2}}{n_{1}}+\frac{\sigma_{L_{2}}^{2}}{n_{0}-n_{1}} .
$$

Now we are searching for minimum of function (7) on $n_{1}$.

The first derivative of function (7) on $n_{1}$ gives us:

$$
\left(\sigma_{T E C_{0}}^{2}\right)_{n}^{\prime}=-\frac{\sigma_{L_{1}}^{2}}{n_{1}}+\frac{\sigma_{L_{2}}^{2}}{\left(n_{0}-n_{1}\right)^{2}} .
$$

Taking into account

$$
\left(\sigma_{T E C_{0}}^{2}\right)_{n}^{\prime}=0
$$

we get:

$$
\frac{n_{1}^{2}}{\sigma_{L_{1}}^{2}}=\frac{\left(n_{0}-n_{1}\right)^{2}}{\sigma_{L_{2}}^{2}} .
$$

From the Formula (9) it is easy to get following square order linear equation:

$$
n_{1}^{2}+2 b n_{1}-b n_{0}=0 \text {. }
$$

where 


$$
b=\frac{n_{0}}{\sigma_{L_{2}}^{2}\left(\frac{1}{\sigma_{L_{1}}^{2}}-\frac{1}{\sigma_{L_{2}}^{2}}\right)} .
$$

Solution of Equation (10) is following:

$$
n_{1}=\sqrt{b^{2}+b n_{0}}-b
$$

Using method of second derivative we can show, that upon solution (12) the total random error reaches its minimum value. The second derivative of Equation (7) is as follows:

$$
\left(\sigma_{T E C_{0}}^{2}\right)_{n}^{\prime \prime}=\frac{2 \sigma_{L_{1}}^{2}}{n_{1}^{3}}+\frac{2 \sigma_{L_{2}}^{2}}{\left(n_{0}-n_{1}\right)^{3}} .
$$

As it can be seen from Formula (13) the second derivative is always positive, which confirm the solution (12).

Therefore, it is shown, that if the limitation is imposed on total number of possible measurements of $T E C_{\rho}$ we should take into account, that the minimum value of total random error of this parameter can be reached upon different numbers of measurements of $\rho_{L_{1}}$ and $\rho_{L_{2}}$.

Due to simmetricity of Formulas (2) and (3) the similar conclusion is true for case of measuring $T E C_{\varphi}$.

\section{Conclusions}

Consequently, it is shown that upon imposed limitation on total number of carried out measurements, due to different values of signal/noise ratios of $L_{1}$ and $L_{2}$, the minimum value of random errors $T E C_{\varphi}$ and $T E C_{\rho}$ can be obtained upon non-equal numbers of measurements of parameters $\rho_{L_{1}}$ and $\rho_{L_{2}}$, and $\varphi_{L_{1}}$ and $\varphi_{L_{2}}$. It is obvious that minimization of total random of $T E C_{\rho}$ and $T E C_{\varphi}$ could lead to an increase of accuracy of resulting value of $T E C_{f}$, which is the main output parameter of the known method [1], designated for calculation of total electron content in ionosphere.

In conclusion we can formulate following results of research carried out:

The analysis of existing method for calculation of total electron content in ionosphere using the GPS occultation measurements does show that due to different values of signal/noise ratios for GPS signals $L_{1}$ and $L_{2}$ the special method of optimal measurements of frequency components of TEC measured by phase and code measurements methods should be developed.

The optimal number of measurements of above said frequency components is determined taking into account the limitation condition imposed on total number of measurements carried out.

\section{References}

[1] Dyrud, L., Jovancevic, A., Brown, A., Wilson, D. and Ganguly, S. (2008) Ionospheric Measurement with GPS: Receiver Techniques and Methods. Radio Science, 43, RS6002.

[2] Chang, Ch-Ch. (1999) Testing on Tropospheric Modelling for GPS Tracking Stations in Taiwan. No 70, 77-94. www.ydu.edu.tw./ cechang/trop_PE.pdf

[3] Grove-Rasmussen, J. (2002) Atmospheric Water Vapour Detection Using Satellite GPS Proofing. Danish Meteorological Institute, Copenhagen. www.dmi.dk/dmi/sr03-05.pdf

[4] Chiu, T.-Ch., Liou, Y.-A., Yeh, W.-H. and Huang, Ch.-Y. (2008) NCURO Data-Retrieval Algorithm in FORMOSAT3 GPS Radio-Occultation Mission. IEEE Transactions on Geoscience and Remote Sensing, 46, 3395-3405. http://dx.doi.org/10.1109/TGRS.2008.2005038

[5] Boniface, K., Aparicio, J.M. and Cardellach, E. (2011) Meteorological Information in GPS-RO Reflected Signals. Atmospheric Measurement Techniques, 4, 1397-1407. http://www.atmos-meas-tech.net/4/1397/2011/amt-4-1397-2011.html

[6] Wee, T.-K. and Kuo, Y.-H. (2013) A Noise-Aware Combination of Dual Frequency Measurements from GPS Radio Occultation. Journal of Geophysical Research: Atmospheres, 118, 12.852-12.868. 
Scientific Research Publishing (SCIRP) is one of the largest Open Access journal publishers. It is currently publishing more than 200 open access, online, peer-reviewed journals covering a wide range of academic disciplines. SCIRP serves the worldwide academic communities and contributes to the progress and application of science with its publication.

Other selected journals from SCIRP are listed as below. Submit your manuscript to us via either submit@scirp.org or Online Submission Portal.
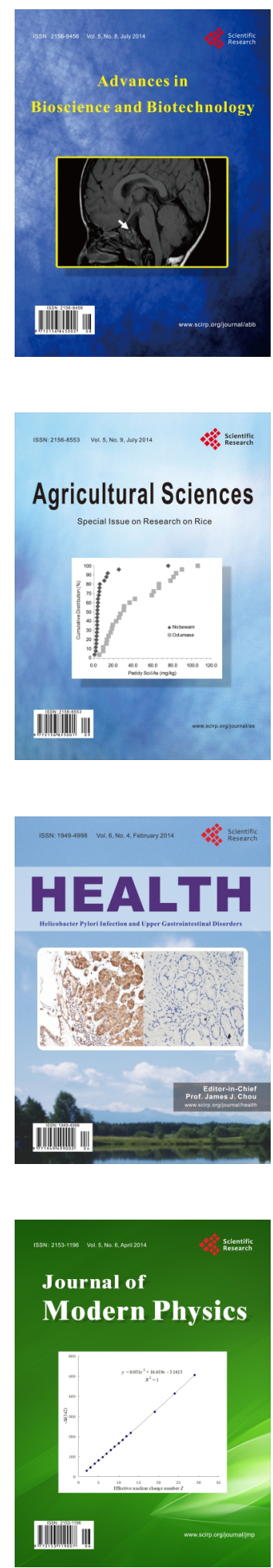
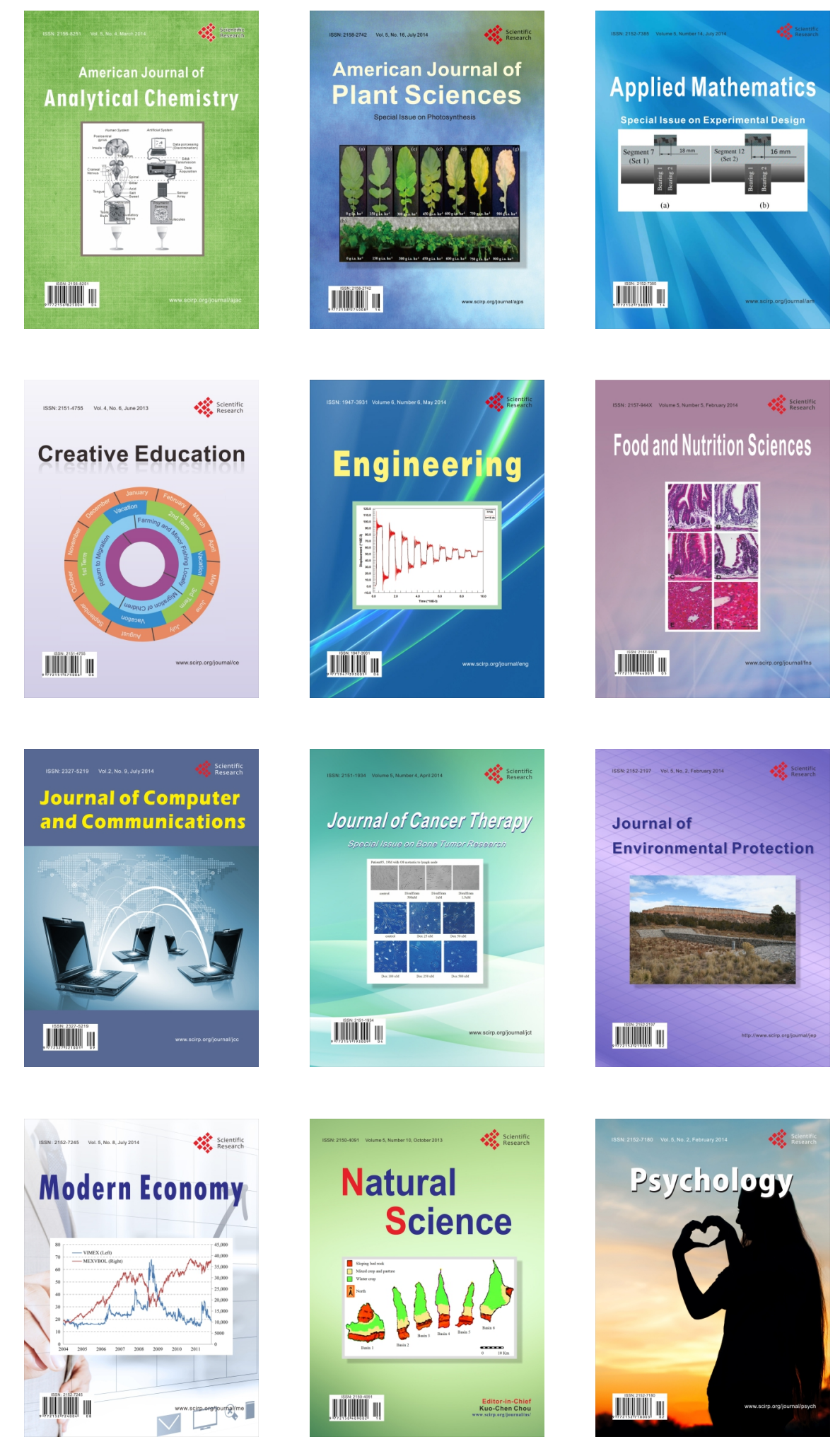